\title{
Comparison of outcomes in patients undergoing rotational atherectomy after unsuccessful coronary angioplasty versus elective rotational atherectomy
}

\author{
Jaroslaw Gorol, Mateusz Tajstra, Bartosz Hudzik, Andrzej Lekston, Mariusz Gąsior \\ Third Department of Cardiology, SMDZ in Zabrze, Silesian Center for Heart Disease, Medical University of Silesia in Katowice, Poland
}

Adv Interv Cardiol 2018; 14, 2 (52): 128-134 DOI: https://doi.org/10.5114/aic.2018.76403

\begin{abstract}
A bstract
Introduction: Revascularization of patients with heavily calcified coronary arteries can be a challenge for interventional cardiologists. The procedural success rate in these patients can be improved by using rotational atherectomy (RA).

Aim: To compare in-hospital outcomes and those at 12-month follow-up between patients who underwent RA as a bailout procedure secondary to failed conventional angioplasty or as an elective procedure.

Material and methods: This is a retrospective analysis of 156 consecutive patients hospitalized at a high-volume percutaneous coronary intervention (PCI) center who underwent RA. In 43 (27.6\%) patients, RA was performed on an elective basis (group 1). In 113 (72.4\%) patients RA was carried out after unsuccessful traditional angioplasty (group 2).

Results: Patients in group 1 more often had a history of peripheral vascular disease (32.6\% vs. 15.9\%; $p=0.03$ ). Group 1 was dominated by patients with multivessel disease $(62.8 \%$ vs. $33.6 \% ; p<0.001)$. The left main coronary artery was more often treated in group 1 ( $25.6 \%$ vs. $2.7 \% ; p<0.001)$. Success rates in the two groups were similar: $93.0 \%$ for group 1 and $91.2 \%$ for group 2 $(p=0.71)$. The rate of in-hospital complications did not significantly differ between the groups. Twelve-month MI, TLR, and TVR rates were similar in both groups. There was no difference in the 12 -month survival rate $(86.1 \%$ vs. $92.0 \%$ in group $2 ; p=0.27)$ or MACE $(16.3 \%$ vs. $15.0 \% ; p=0.8)$

Conclusions: Rotational atherectomy is associated with high efficacy and a relatively low risk of complications, with no significant differences in outcomes between patients treated with primary and secondary RA procedures.
\end{abstract}

Key words: coronary artery disease, rotablation, calcifications.

\section{Introduction}

Advances in medicine, particularly in the diagnosis and treatment of cardiovascular diseases, have contributed to the increase in the number of elderly patients referred to cardiologists. Coronary artery calcification increases with age, and among patients undergoing percutaneous coronary intervention $(\mathrm{PCl})$, it is present in about $30 \%$ of cases on angiographic assessment, and up to $70 \%$ of cases on intravascular ultrasound [1].

Revascularization in patients with heavily calcified coronary arteries can pose a challenge for interventional cardiologists. Calcified lesions often create problems during $\mathrm{PCl}$ and require the use of more aggressive techniques, typically associated with a greater risk of complications [2, 3]. The procedural success rate in these patients can be improved by using rotational atherectomy
(RA), which is a technique that modifies the atherosclerotic plaque and facilitates stent delivery and implantation. The European Society of Cardiology guidelines on coronary revascularization, based on the ROTAXUS (Rotational Atherectomy Prior to Taxus Stent Treatment for Complex Native Coronary Artery Disease) study, discuss the possibility of using RA in patients with significantly stenosed and calcified lesions but recommend against the routine use of this method $[4,5]$. Rotational atherectomy can be performed electively or as a "bailout" procedure after unsuccessful angioplasty.

\section{Aim}

Few published reports have compared the outcomes of RA after unsuccessful angioplasty and RA performed electively. Therefore, the aim of this study was to com-

\section{Corresponding author:}

Jaroslaw Gorol MD, Third Department of Cardiology, SMDZ in Zabrze, Silesian Center for Heart Disease, Medical University of Silesia, 9 Curie-Sklodowskiej St, 41-800 Katowice, Poland, phone: +48 79299 47 93, e-mail: jaroslawgorol@interia.pl

Received: 25.12.2017, accepted: 26.02.2018. 
pare early and late outcomes in patients who underwent RA as a bailout or elective procedure.

\section{Material and methods}

We analyzed 156 consecutive patients hospitalized at a high-volume PCl center between 2009 and 2016 who underwent RA. All patients were included in an ongoing, prospective registry of RA procedures.

In 43 (27.6\%) patients designated as group 1, RA was performed on an elective basis (primary RA). Elective RA was defined as the initial use of RA without previous device failure. In 113 (72.4\%) patients designated as group 2, RA was carried out after unsuccessful traditional angioplasty (inability to cross the lesion with a balloon or stent $-25.7 \%$, balloon underexpansion $-74.3 \%$ ) - secondary RA.

Coronary angiography was performed according to the standard institutional protocol. The degree of calcification was classified according to the Mintz criteria as mild, moderate, or severe [3]. The decision to perform elective RA or conventional angioplasty was left to the discretion of the operator and was usually based on the angiographic appearance of the target lesion. Heavy calcifications or effects of $\mathrm{PCl}$ previously performed on other lesions most likely influenced the operator's decision. Selection bias between the primary and secondary RA strategy often depended on operator experience. However, as this is a retrospective study, we do not have full data explaining the operator's decision.

To prevent bradyarrhythmias, a temporary electrode was inserted into the right ventricle before carrying out $\mathrm{RA}$ in the right coronary artery (RCA), the circumflex branch (in the case of a dominant left coronary arterial system), or in selected cases of multivessel coronary artery disease. The procedure was performed using a Rotablator system (Boston Scientific Scimed, Maple Grove, MN, US) with a burr size ranging from 1.25 to $2.25 \mathrm{~mm}$. Based on current evidence, the burr diameter was selected so as to achieve a burr/artery ratio $<0.7[6,7]$. The rotation speed of the burr during RA ranged between 130000 and 150000 rpm. Short (10-25-second) sessions of RA were preferred. All patients without contraindications received drug-eluting stents (DES). Procedural success was defined as successful stent implantation with residual stenosis of less than $20 \%$ diameter and TIMI-3 flow. Periprocedural myocardial infarction (MI) was diagnosed according to the current recommendations [8]. All patients received dual antiplatelet therapy (aspirin and clopidogrel, ticagrelor, or prasugrel), which was then continued for at least 12 months after DES implantation and 4 weeks after implantation of a bare metal stent (BMS). During the procedure, anticoagulant therapy (unfractionated heparin) was administered, and use of Ilb/IIla inhibitors was left to the discretion of the operator. Quantitative coronary angiography (QCA) of the lesions was performed by two independent interventional cardiologists.

Patients' demographic characteristics, medical history, and clinical data were retrieved from the electronic patient record database at our institution. The following variables were analyzed: cardiovascular risk factors, angiographic characteristics, and type of equipment used during the procedure.

The primary endpoint was in-hospital and 12-month all-cause mortality. Secondary endpoints included myocardial infarction, the need for repeat target vessel revascularization (TVR) or revascularization of the treated lesion (TLR), stroke, and all major adverse cardiovascular events (MACE), which consisted of death, MI, TVR, TLR, and stroke. The follow-up data were obtained from the official National Health Fund database as well as from direct phone calls to patients or their families. We did not obtain data on cause of death.

\section{Statistical analysis}

Continuous variables are presented as mean and standard deviation and compared using Student's $t$-test and Mann-Whitney $U$-test. Categorical variables are presented as numbers and percentages and compared using the $\chi^{2}$ test. Twelve-month mortality was analyzed with Kaplan-Meier curves and compared using the log-rank test. The logistic regression method was used to determine independent predictors of 12-month mortality. Multivariate models were constructed by including all univariate predictors with a $p$-value $<0.2$. Nonsignificant covariates were removed from the model in a backward stepwise fashion until all variables left in the model were statistically significant.

\section{Results}

Patients in the two groups did not differ with regard to sex, age, or cardiovascular risk factors. Patients undergoing RA after unsuccessful angioplasty (group 2) more often had a history of previous coronary interventions. The baseline characteristics of both groups are listed in Table I. Patients in group 1 more often had a history of peripheral artery disease. Mean EuroSCORE II was higher in group 1. Group 1 was dominated by patients with multivessel coronary artery disease. There were more ostial, bifurcation and longer lesions treated in group 1 (Table II). In both groups, RA was performed most frequently in the RCA, while left main coronary artery (LMCA) RA was performed more often in the elective group. As most of the RA procedures were performed on the RCA, a temporary electrode was implanted in $53.2 \%$ of patients and the rate was similar in both groups. There were no complications related to electrode implantation. The success rates in the two groups were similar.

The rate of in-hospital complications did not differ significantly between groups. There was one case 
Table I. Baseline clinical characteristics

\begin{tabular}{|c|c|c|c|}
\hline Parameter & Elective RA $(n=43)$ & Bailout RA $(n=113)$ & $P$-value \\
\hline Male & $27(62.8 \%)$ & $74(65.5 \%)$ & 0.75 \\
\hline Age [years] & $71.0 \pm 8.2$ & $69.0 \pm 8.7$ & 0.71 \\
\hline Hypertension & $39(91.2 \%)$ & $95(84.1 \%)$ & 0.2 \\
\hline Diabetes & $21(48.8 \%)$ & $57(50.4 \%)$ & 0.88 \\
\hline Diabetes on insulin & $9(20.93 \%)$ & $20(17.7 \%)$ & 0.65 \\
\hline Dyslipidaemia & $33(76.7 \%)$ & $81(71.7 \%)$ & 0.48 \\
\hline Chronic kidney disease eGFR $<60 \mathrm{ml} / \mathrm{min} / 1.73 \mathrm{~m}^{2}$ & $10(23.3 \%)$ & $28(24.8 \%)$ & 0.84 \\
\hline Atrial fibrillation & $13(30.9 \%)$ & $25(23.2 \%)$ & 0.56 \\
\hline Peripheral vascular disease & $14(32.6 \%)$ & $18(15.9 \%)$ & 0.03 \\
\hline EuroSCORE 2 mean & $5.9 \pm 6.9$ & $4.6 \pm 7.0$ & 0.02 \\
\hline History of stroke & $4(9.3 \%)$ & $13(11.5 \%)$ & 0.7 \\
\hline Smoker & $11(26.19 \%)$ & $30(28.04 \%)$ & 0.98 \\
\hline Obesity & $17(39.5 \%)$ & $41(36.3 \%)$ & 0.74 \\
\hline Body mass index, mean \pm SD & $29.12 \pm 5.4$ & $28.88 \pm 4.47$ & 0.21 \\
\hline Family history of CAD & $15(35.7 \%)$ & $40(37.4 \%)$ & 0.99 \\
\hline Previous MI & $27(62.8 \%)$ & $77(68.1 \%)$ & 0.60 \\
\hline Previous PCl & $22(51.2 \%)$ & $84(74.3 \%)$ & 0.006 \\
\hline Previous CABG & $10(23.3 \%)$ & $29(25.7 \%)$ & 0.7 \\
\hline ACS (UA + NSTEMI) & $14(32.6 \%)$ & $31(27.4 \%)$ & 0.58 \\
\hline Ejection fraction, mean \pm SD [\%] & $42.5 \pm 11.0$ & $44.9 \pm 10.7$ & 0.84 \\
\hline Ejection fraction $<35 \%$ & $8(18.6 \%)$ & $22(19.5 \%)$ & 0.84 \\
\hline Hospitalization time, mean \pm SD [days] & $11.05 \pm 8.82$ & $8.61 \pm 5.68$ & 0.05 \\
\hline RA during the same procedure & $11(25.6 \%)$ & $15(13.3 \%)$ & 0.65 \\
\hline Delayed RA & $32(74.4 \%)$ & $98(86.7 \%)$ & 0.17 \\
\hline
\end{tabular}

$C A D$ - coronary artery disease, $M I$ - myocardial infarction, $P C I$ - percutaneous coronary intervention, CABG - coronary artery bypass grafting, UA - unstable angina, NSTEMI - non-ST elevation myocardial infarction, $R A$ - rotational atherectomy.

of in-hospital death in group 1 (2 days after the procedure). The incidence of periprocedural MI was similar in both groups. One patient in group 1 was diagnosed with ischemic stroke 1 day after the procedure (Table III). There was no difference in the 12-month survival rate ( $86.1 \%$ vs. $92.0 \%, p=0.27$; Figure 1 ), and the secondary endpoints also occurred with similar frequency in both groups (Table III).

Independent risk factors of all-cause mortality following RA were: left ventricular ejection fraction $<35 \%$, creatinine level at discharge and age (Table IV).

\section{Discussion}

Coronary calcifications are often a part of systematic vessel calcinosis [9]. The treatment of calcified coronary lesions is associated with a high risk of restenosis and TLR [10]. Rotational atherectomy represents an alternative for patients with calcified arteries. Since its development nearly 30 years ago, the idea of an isolated RA [11] was quickly abandoned; however, in the DES era this method has seen a resurgence of interest as it has been associated with significantly reduced restenosis rates $[12,13]$. Rotational atherectomy is often performed electively, but in everyday clinical practice RA is more widely performed as a secondary treatment after unsuccessful conventional angioplasty. The results, in the context of their clinical implications, arising from the present study are, first, that more than $70 \%$ of patients treated with RA at a center with extensive experience in interventional treatment underwent the procedure secondary to an ineffective attempt at conventional $\mathrm{PCl}$; secondly, RA was performed in patients considered to be high risk in the context of clinical as well as angiographic characteristics; thirdly, RA as a technique demonstrated high efficacy and was associated with relatively few complications; fourthly, there were no significant differences in outcomes at 1 year after the procedure between patients who underwent RA after unsuccessful PCI and those who underwent RA electively.

Recently, several studies have reported the outcomes of DES implantation preceded by RA. Our patient cohort seems to represent a higher-risk group than previously 
Table II. Angiographic features

\begin{tabular}{|c|c|c|c|}
\hline Parameter & Elective RA $(n=43)$ & Bailout RA $(n=113)$ & $P$-value \\
\hline Three-vessel disease & $27(62.8 \%)$ & $38(33.6 \%)$ & 0.001 \\
\hline Left main RA & $11(25.6 \%)$ & $3(2.7 \%)$ & $<0.001$ \\
\hline RCA RA & $18(41.9 \%)$ & $61(54.0 \%)$ & 0.18 \\
\hline ACC AHA classification lesion type B/C & $43(100.0 \%)$ & $113(100.0 \%)$ & - \\
\hline Severe calcifications & $41(95.3 \%)$ & $94(83.1 \%)$ & 0.15 \\
\hline Bifurcation lesion & $14(32.6 \%)$ & $10(8.9 \%)$ & 0.001 \\
\hline Chronic total occlusion & 0 & $5(4.42 \%)$ & 0.32 \\
\hline Ostial lesion & $15(34.9 \%)$ & $19(18.8 \%)$ & 0.02 \\
\hline Lesion length, mean $\pm \mathrm{SD}[\mathrm{mm}]$ & $31.2 \pm 24.1$ & $28.9 \pm 18.6$ & 0.04 \\
\hline Lesion length > $20 \mathrm{~mm}$ & $22(51.2 \%)$ & $66(58.4 \%)$ & 0.34 \\
\hline \% stenosis in QCA & $76.0 \pm 9.3$ & $79.2 \pm 9.8$ & 0.75 \\
\hline $\mathrm{MLD}[\mathrm{mm}]$ & $0.77 \pm 0.37$ & $0.62 \pm 0.33$ & 0.32 \\
\hline Reference diameter [mm] & $3.24 \pm 0.64$ & $2.98 \pm 0.51$ & 0.02 \\
\hline Temporary electrode in right ventricle & $24(55.8 \%)$ & $59(52.2 \%)$ & 0.75 \\
\hline Femoral approach & $35(81.4 \%)$ & $101(89.4 \%)$ & 0.18 \\
\hline 7-French guiding catheter & $27(62.8 \%)$ & $83(73.5 \%)$ & 0.39 \\
\hline Burr size in first step, mean $\pm \mathrm{SD}[\mathrm{mm}]$ & $1.68 \pm 0.38$ & $1.57 \pm 0.25$ & 0.21 \\
\hline No. of RA sessions & $4.0 \pm 1.41$ & $3.93 \pm 1.72$ & 0.16 \\
\hline No. of burrs, mean \pm SD & $1.40 \pm 0.49$ & $1.32 \pm 0.47$ & 0.64 \\
\hline$>1$ burr & $17(39.5 \%)$ & $36(38.1 \%)$ & 0.37 \\
\hline Burr-to-artery ratio, mean \pm SD & $0.52 \pm 0.098$ & $0.54 \pm 0.094$ & 0.56 \\
\hline Predilatation & $42(97.7 \%)$ & $113(100 \%)$ & 0.96 \\
\hline Number of stents, mean \pm SD & $1.86 \pm 1.03$ & $1.92 \pm 1.03$ & 0.52 \\
\hline Total stent length $[\mathrm{mm}]$ & $47.84 \pm 25.71$ & $43.26 \pm 23.69$ & 0.33 \\
\hline Drug-eluting stent & $40(93.0 \%)$ & $102(90.3 \%)$ & 0.73 \\
\hline Postdilatation & $38(88.4 \%)$ & $84(79.2 \%)$ & 0.12 \\
\hline GP IIb/IIla inhibitor & $6(14.0 \%)$ & $13(11.5 \%)$ & 0.35 \\
\hline Procedure time [min] & $146.34 \pm 40.17$ & $135.47 \pm 46.15$ & 0.09 \\
\hline Fluoroscopy time [min] & $35.59 \pm 17.78$ & $36.24 \pm 17.61$ & 0.76 \\
\hline Radiation dose [Gy] & $2.26 \pm 1.04$ & $2.33 \pm 1.38$ & 0.71 \\
\hline Final TIMI-3 flow & $41(95.4 \%)$ & $109(96.5 \%)$ & 0.75 \\
\hline MLD after procedure [mm] & $3.08 \pm 0.71$ & $2.89 \pm 0.62$ & 0.27 \\
\hline Acute lumen gain, mean [mm] & $2.31 \pm 0.63$ & $2.27 \pm 0.61$ & 0.78 \\
\hline Procedural success & $40(93.0 \%)$ & $103(91.2 \%)$ & 0.71 \\
\hline
\end{tabular}

$R A$ - rotational atherectomy, $Q C A$ - quantitative coronary angiography, $M L D$ - minimal lumen diameter.

reported, especially with regard to the presence of low ejection fraction and history of MI or coronary artery bypass grafting (CABG). Compared to the study of Clavijo et al. or Abdel-Wahab et al., our cohort included higher proportion(s) of patients with diabetes or a history of MI, and we also attempted to treat longer lesions in coronary arteries $[14,15]$. Similarly, the ROTATE study also included fewer patients with diabetes mellitus, low ejection fraction, or previous $\mathrm{MI}, \mathrm{PCl}$, or CABG [16].

Despite the inclusion of high-risk patients, the effectiveness of RA in our cohort was good. The success rate of the procedure has been reported to range from 90 to $100 \%$. Our success rate was in this range. Of note, our study had no exclusion criteria. In some previous studies, performing RA without stent implantation was an exclusion criterion, while in our study stent implantation was required. We adopted restrictive criteria for treatment effectiveness, using a residual stenosis threshold lower than that of other studies such as those reported by Naito, Abdul-Wahab, and Furuichi, which accepted residual stenosis < $30 \%[12,15,17]$. The procedural success rate in our registry is comparable to that from the ROTATE reg- 
Table III. Rate of in-hospital and 12-month adverse events

\begin{tabular}{|c|c|c|c|}
\hline Parameter & Elective RA $(n=43)$ & Bailout RA $(n=113)$ & $P$-value \\
\hline \multicolumn{4}{|l|}{ In-hospital complications: } \\
\hline Dissection & $2(4.7 \%)$ & $4(3.5 \%)$ & 0.67 \\
\hline Slow flow/no flow & $2(4.7 \%)$ & $4(3.5 \%)$ & 0.67 \\
\hline Rotawire fracture & 0 & $1(0.9 \%)$ & 0.54 \\
\hline Perforation & 0 & $2(1.8 \%)$ & 0.93 \\
\hline Access site complications (hematoma, pseudoaneurysm) & $5(11.6 \%)$ & $4(3.9 \%)$ & 0.20 \\
\hline Periprocedural MI & $2(4.7 \%)$ & $5(4.4 \%)$ & 0.95 \\
\hline Stroke & 0 & $1(0.9 \%)$ & 0.54 \\
\hline MACE & $3(7.0 \%)$ & $6(5.3 \%)$ & 0.69 \\
\hline Death & $1(2.3 \%)$ & 0 & 0.10 \\
\hline \multicolumn{4}{|l|}{ 12-month complications: } \\
\hline TLR & $1(2.3 \%)$ & $6(5.3 \%)$ & 0.42 \\
\hline TVR & $2(4.7 \%)$ & $8(7.1 \%)$ & 0.58 \\
\hline $\mathrm{MI}$ & $2(4.7 \%)$ & $8(7.1 \%)$ & 0.58 \\
\hline Stroke & 0 & $1(0.9 \%)$ & 0.54 \\
\hline MACE & $7(16.3 \%)$ & $17(15.0 \%)$ & 0.8 \\
\hline Death & $6(14.0 \%)$ & $9(8.0 \%)$ & 0.27 \\
\hline
\end{tabular}

$R A$ - rotational atherectomy, MI-myocardial infarction, TLR - target lesion revascularization, TVR-target vessel revascularization, MACE - major adverse cardiac events.

Table IV. Independent predictors of 12-month mortality

\begin{tabular}{lcccc} 
Variable & Hazard ratio & 95\% confidence interval & Multivariate analysis ( $\boldsymbol{p}$-value) \\
\hline ACS on admission & 2.795 & $1.065-6.339$ & 0.07 \\
\hline Arterial hypertension & 0.131 & $0.056-1.776$ & 0.191 \\
\hline LVEF $<35 \%$ & 3.287 & $1.540-7.018$ & 0.02 & 0.021 \\
\hline Creatinine level & 1.016 & $1.002-1.029$ & 0.019
\end{tabular}

ACS - acute coronary syndrome, LVEF - left ventricular ejection fraction.

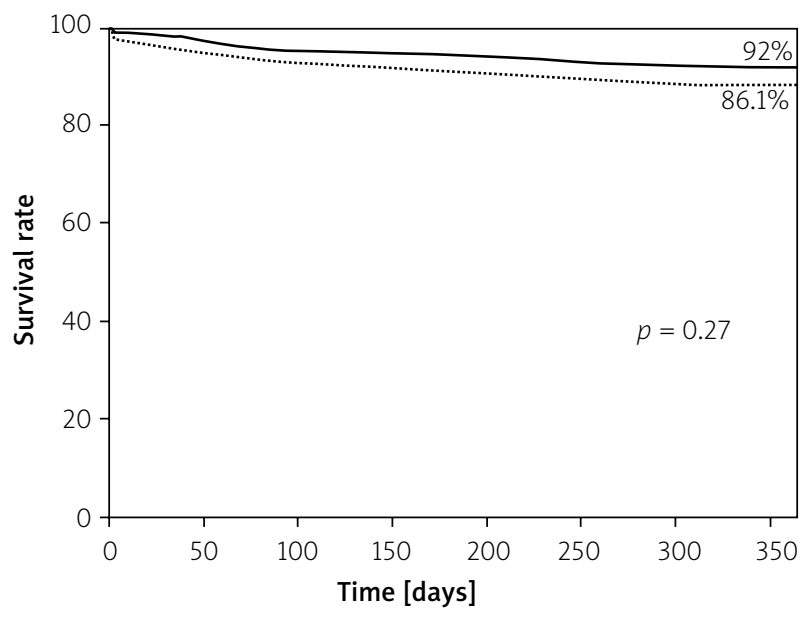

_ Non-elective RA …..... Elective RA

Figure 1. Kaplan-Meier 12-month survival curves in patients undergoing elective RA and RA after unsuccessful PCI istry (90.6\%). Similar to the present study, in a comparison of 50 patients with severely calcified lesions who underwent RA at Zurich University Hospital, of whom 26 underwent elective rotablation due to the presence of severe calcifications on coronary angiography and 24 underwent rotablation secondary to failed conventional coronary intervention, there was no difference in efficacy between the elective and secondary RA groups [18].

The overall procedural complication rate in the present study also compares favorably with data reported in the literature. The dissection rate was lower than that reported in some previous studies [15]. The perforation rate was low and comparable to that presented in the Polish Registry [19]. Also the rate of in-hospital MI was relatively low compared to previous reports. The predominant use of femoral access causes frequent local complications but did not result in significant differences compared to other studies [14, 15]. We experienced one in-hospital death, as has been reported in some other studies [15]. 
The observed mortality rate at 12 months in the present study is similar to the rates reported in other published series of patients treated with RA. For example, Garcia de Lara et al. reported mortality rates at 12-month follow-up of $10 \%$ and $14 \%$ in the presence of MI, which is higher than in our registry [20]. In comparison to the ROTATE study, our cohort had a similar rate of MACE, but we also included stroke and TVR as endpoints. The incidence of MACE in the present study is lower than in the ROTAXUS study. Despite the high rate of angiographic success in the ROTAXUS cohort at 2-year follow-up, almost one-third of those patients experienced MACE, regardless of the treatment strategy $[4,21]$. Of note, in the ROTAXUS study, patients with low ejection fraction, acute coronary syndrome, and unprotected left main disease were excluded. These differences allow us to conclude that the population of patients undergoing RA from our "all-comers" registry is more consistent with clinical reality than the randomized trial. It should be noted that in the ROTAXUS study, crossover to RA was necessary in $12.5 \%$ of patients in the group randomized to standard balloon dilatation before stenting. Thus, although the ROTAXUS study did not support the use of elective $\mathrm{RA}$, the present analysis suggests that there is a need for further studies comparing primary and secondary RA in different patient populations.

Independent risk factors of all-cause mortality following RA were: left ventricular ejection fraction $<35 \%$, creatinine level at discharge and age. Impaired LVEF and renal disease were also independent risk factors of allcause mortality in another study [22].

In our study the elective group had worse baseline and lesion characteristics such as higher EuroSCORE, higher prevalence of peripheral vascular disease, three-vessel disease, left main disease, bifurcation and ostial lesions, which probably caused the longer procedural time and lower survival rate. In the absence of comorbidities, most patients from the elective group would probably be referred for $C A B G$ due to multivessel and left main disease.

There are few reports comparing elective RA and RA after unsuccessful $\mathrm{PCl}$. Compared to the previous study performed at Zurich University Hospital, the present study analyzed similar clinical characteristics but included more patients with a history of $\mathrm{MI}, \mathrm{PCl}$, or CABG and also did not find any significant differences between primary and secondary RA in terms of in-hospital and long-term outcomes. That previous study had better long-term results but the follow-up period was 6 months instead of 12. Compared to another study that enrolled 62 consecutive patients undergoing RA after ineffective conventional $\mathrm{PCl}$, we observed better $\mathrm{MI}$ and MACE rates in the hospital and at follow-up [23].

In a different study whose aim was to compare outcomes of bailout and planned rotational atherectomy, 667 patients from the ROTATE study were analyzed. The main findings of this study were as follows: in-hospital outcomes were comparable between the two groups; the incidence of MACE at 1 year was significantly higher in the planned RA group amongst the unadjusted population with the differences between groups less marked after propensity-score adjustment; and the planned RA group benefited with regards to procedural outcomes with a reduction of procedural and fluoroscopy times, and number of predilation balloon catheters used [24].

A registry performed by Allali et al. compared primary and secondary RA. They concluded that elective RA was linked to a shortened RA procedural duration and a reduction of the rate of coronary dissections in selected lesions. However, this strategy does not influence longterm clinical outcomes. Similarly, we had a similar success rate and long-term outcomes. Noteworthy, our study population had a less favorable clinical profile with a high rate of previous $\mathrm{PCls}$ [25].

Based on of these results, the elective and secondary RA strategy appears to be both feasible and safe, and should be considered as an option of treatment for calcified lesions. We are in need of further studies since there is little information on procedural, in-hospital, and longterm outcomes of bailout RA as compared to an elective strategy for complex calcified coronary lesions.

The present study has some limitations. It is a retrospective study, enrolling patients from a single center, and there is no control group. The study groups were relatively small, and the results need to be confirmed in a larger population or in a randomized trial.

\section{Conclusions}

The analyzed data from our center indicate that RA treatment is associated with high efficacy and a relatively low risk of complications, and there is no advantage to either elective RA or RA after unsuccessful angioplasty at 12-month follow-up.

\section{Conflict of interest}

The authors declare no conflict of interest.

\section{References}

1. Mintz GS, Popma JJ, Pichard AD et al. Patterns of calcification in coronary artery disease. A statistical analysis of intravascular ultrasound and coronary angiography in 1155 lesions. Circulation 1995; 91: 1959-65.

2. Gilutz H, Weinstein JM, Ilia R. Repeated balloon rupture during coronary stenting due to a calcified lesion: an intravascular ultrasound study. Catheter Cardiovasc Interv 2000; 50: 212-4.

3. Pershad A, Buchbinder M. Management of calcified lesions in 2004. Int J Cardiovasc Intervent 2005; 7: 199-204.

4. Abdel-Wahab M, Richardt G, Joachim Büttner $\mathrm{H}$, et al. Highspeed rotational atherectomy before paclitaxel-eluting stent implantation in complex calcified coronary lesions: the randomized ROTAXUS (Rotational Atherectomy Prior to Taxus Stent Treatment for Complex Native Coronary Artery Disease) trial. JACC Cardiovasc Interv 2013; 6: 10-9. 
5. Windecker S, Kolh P, Alfonso F, et al. 2014 ESC/EACTS Guidelines on myocardial revascularization: The Task Force on Myocardial Revascularization of the European Society of Cardiology (ESC) and the European Association for Cardio-Thoracic Surgery (EACTS). Eur Heart J 2014; 35: 2541-619.

6. Safian RD, Feldman T, Muller DWM, et al. Coronary Angioplasty and Rotablator Atherectomy Trial (CARAT): Immediate and late results of a prospective multicenter randomized trial. Catheter Cardiovasc Interv 2001; 53: 213-20.

7. Whitlow PL, Bass TA, Kipperman RM, et al. Results of the study to determine rotablator and transluminal angioplasty strategy (STRATAS). Am J Cardiol 2001; 87: 699-705.

8. Thygesen K, Alpert JS, Jaffe AS, et al. Third universal definition of myocardial infarction. Eur Heart J 2012; 33: 2551-67.

9. Nonin S, Iwata S, Sugioka K, et al. Plaque surface irregularity and calcification length within carotid plaque predict secondary events in patients with coronary artery disease. Atherosclerosis 2017; 256: 29-34.

10. Moses JW, Carlier S, Moussa I. Lesion preparation prior to stenting. Rev Cardiovasc Med 2004; 5 (suppl 2): 16-21.

11. Reifart N, Vandormael M, Krajcar M, et al. Randomized comparison of angioplasty of complex coronary lesions at a single center. Excimer Laser, Rotational Atherectomy, and Balloon Angioplasty Comparison (ERBAC) Study. Circulation 1997; 96: 91-8.

12. Naito R, Sakakura K, Wada $H$, et al. Comparison of long-term clinical outcomes between sirolimus-eluting stents and paclitaxel-eluting stents following rotational atherectomy. Int Heart J 2012; 53: 149-53.

13. Benezet J, Díaz de la Llera LS, Cubero JM, et al. Drug-eluting stents following rotational atherectomy for heavily calcified coronary lesions: long-term clinical outcomes. J Invasive Cardiol 2011; 23: 28-32.

14. Clavijo LC, Steinberg DH, Torguson R, et al. Sirolimus-eluting stents and calcified coronary lesions: clinical outcomes of patients treated with and without rotational atherectomy. Catheter Cardiovasc Interv 2006; 68: 873-8.

15. Abdel-Wahab M, Baev R, Dieker P, et al. Long-term clinical outcome of rotational atherectomy followed by drug-eluting stent implantation in complex calcified coronary lesions. Catheter Cardiovasc Interv 2013; 81: 285-91.

16. Kawamoto H, Latib A, Ruparelia N, et al. In-hospital and midterm clinical outcomes of rotational atherectomy followed by stent implantation: the ROTATE multicentre registry. Eurolntervention 2016; 12: 1448-56.

17. Furuichi S, Sangiorgi GM, Godino C, et al. Rotational atherectomy followed by drug-eluting stent implantation in calcified coronary lesions. Eurolntervention 2009; 5: 370-4.

18. Puck M, Regli C, Toggweiler S, et al. Rotational atherectomy of severely calcified coronary artery lesions: experience at Zurich University Hospital. Cardiovasc Med 2010; 13: 81-5.

19. Januszek R, Siudak Z, Dziewierz A, et al. Predictors of in-hospital effectiveness and complications of rotational atherectomy (from the ORPKI Polish National Registry 2014-2016). Catheter Cardiovasc Interv 2017 in press doi: 10.1002/ccd.27372.

20. García de Lara J, Pinar E, Ramón Gimeno J, et al. Percutaneous coronary intervention in heavily calcified lesions using rotational atherectomy and paclitaxel-eluting stents: outcomes at one year. Rev Esp Cardiol 2010; 63: 107-10.

21. de Waha S, Allali A, Büttner HJ, et al. Rotational atherectomy before paclitaxel-eluting stent implantation in complex calcified coronary lesions: two-year clinical outcome of the randomized ROTAXUS trial. Catheter Cardiovasc Interv 2016; 87: 691-700.

22. Édes IF, Ruzsa Z, Szabó G, et al. Clinical predictors of mortality following rotational atherectomy and stent implantation in high-risk patients: a single center experience. Catheter Cardiovasc Interv 2015; 86: 634-41.

23. Wojtczak $M$, Kubler P, Ferenc $M$, et al. Coronary rotational atherectomy in patients with unsuccessful classical angioplasty - in-hospital and six-month follow-up. Postep Kardiol Interw 2012; 8: 85-90.

24. Kawamoto H, Latib A, Ruparelia N, et al. Planned versus provisional rotational atherectomy for severe calcified coronary lesions: insights From the ROTATE multi-center registry. Catheter Cardiovasc Interv 2016; 88: 881-9.

25. Allali A, Abdel-Wahab M, Sulimov DS, et al. Comparison of bailout and planned rotational atherectomy for heavily calcified coronary lesions: a single-center experience. J Interv Cardiol 2017; 30: 124-33. 